\title{
High frequency optogenetic activation of inputs to the lateral amygdala forms distant association with foot-shock
}

\author{
Fei Li ${ }^{1}$, Chun-Hui Jia ${ }^{1 \dagger}$, Jun Huang ${ }^{1 \dagger}$, Guo-Qiang Bi ${ }^{12^{*}}$ and Pak-Ming Lau ${ }^{1 *}$
}

\begin{abstract}
Aim: A hallmark of classical conditioning is that conditioned stimulus (CS) must be tightly coupled with unconditioned stimulus (US), often requiring temporal overlap between the two, or a short gap of several seconds. In this study, we investigate the temporal requirements for fear conditioning association between a strong artificial CS, high-frequency optogenetic activation of inputs into the lateral amygdala of rats, and a foot-shock to the animal with delays up to many minutes.

Methods: AAV-oChIEF-tdTomato viruses were injected into the auditory cortex and the medial geniculate nucleus of rats. An optical fiber was implanted just above the lateral amygdala of the animal. Optogenetic high-frequency stimuli (oHFS; containing five 1-s trains of $100 \mathrm{~Hz}$ laser pulses) were delivered to the lateral amygdala, before or after (with varying intervals) a foot-shock that elicits fear responses in the animal. Pre-trained lever-press behavior was used to assess the degree of fear recall by optogenetic test stimuli (OTS; $10 \mathrm{~Hz}$ for $2 \mathrm{~min}$ ) $24 \mathrm{~h}$ after the association experiment.

Results: In contrast to the tight temporal requirement for classical conditioning with paired optogenetic moderatefrequency stimuli (oMFS; $10 \mathrm{~Hz}$ for $20 \mathrm{~s}$ ) and foot-shock, oHFS followed by foot-shock with a 5-min or even 1-h (but not 3-h) interval could successfully establish an association to be recalled by OTS the next day. Meanwhile, foot-shock followed by oHFS with a 5-min (but not 1-h) interval could also establish the conditioning. Thus, distant association may be formed between temporally distant stimuli when the CS is strong.
\end{abstract}

Keywords: Lateral amygdala, Optogenetic stimulation, Distant fear conditioning

Animals live in a complex environment and experience many events of different significance every day. Learning the causal or temporal relationship, i.e., forming association, between important events is crucial for the animal to adapt to the environment and survive. In classical conditioning, long-term associative memory is formed after repeated pairing of the conditioned stimulus (CS) and the unconditioned stimulus (US) [1, 2], often with

\footnotetext{
* Correspondence: gqbi@ustc.edu.cn; plau@ustc.edu.cn

${ }^{+}$Chun-Hui Jia and Jun Huang contributed equally to this work.

'CAS Key Laboratory of Brain Function and Disease, and School of Life

Sciences, University of Science and Technology of China, Hefei 230026, Anhui, China

Full list of author information is available at the end of the article
}

the US presented before the end the CS presentation as in the case of delay conditioning, or with the US following the CS by short gaps of several seconds as in the case of trace conditioning [3, 4]. When the gap is too long, e.g. beyond $30 \mathrm{~s}$, the mild CS and the strong US are considered "unpaired", and association cannot be formed between them [4]. Intuitively, the significance of sequential events could also have an impact on the formation of association. However, it has remained unclear whether a stronger (and perhaps more significant) "CS"like stimulus can form "distant" association with the US separated by longer gaps.

The lateral amygdala (LA) has been established as a key brain area for auditory fear conditioning [5-7]. In a 


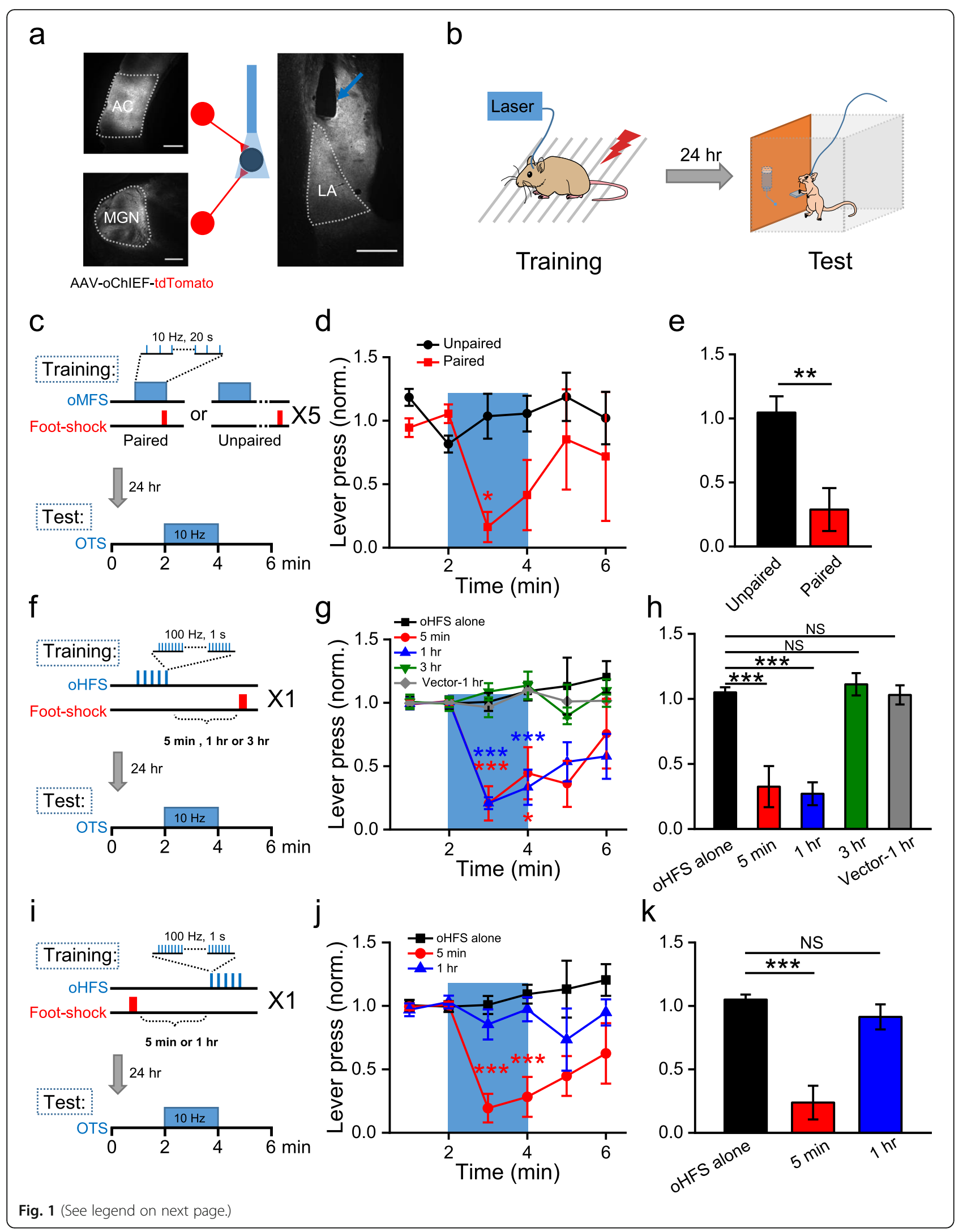




\section{(See figure on previous page.)}

Fig. 1 Association between optogenetic stimulation and foot-shock. a Expression of oChIEF-tdTomato in the AC and the MGN (left), as well as the LA (right) 4 weeks after viral injection. Blue arrow indicates position of implanted optic fiber. Scale bars: $500 \mu \mathrm{m}$. $\mathbf{b}$ Behavioral paradigms of associative fear training (left) and recall test (right). c. Fear training using oMFS paired or unpaired with foot-shocks (repeated every 3 min for 5 times). Fear response was assessed in the lever-pressing box $24 \mathrm{~h}$ later with OTS. $\mathbf{d}$ Normalized number of lever presses (shown in 1-min bin) from lever-pressing tests $24 \mathrm{~h}$ after fear training. Lever pressing was significantly inhibited by OTS (blue area) in the paired group ( $n=4)$ but not in the unpaired group $(n=4) .{ }^{*}$ indicates $P<0.05$; one-way repeated ANOVA followed by Tukey's multiple comparison test. All error bars in this and subsequent figures are SEM. e Mean normalized number of lever presses during the 2 min of the OTS. Significant reduction in lever pressing was found in paired group $(n=4)$ compared with the unpaired group $(n=4)$. ${ }^{* *}$ indicates $P<0.01$; Student's t-test. $\mathbf{f}$ - $\mathbf{h}$ Similar to $\mathbf{c}$-e, but for fear training with oHFS followed by a 3-s foot-shock with a delay of $5 \mathrm{~min}, 1 \mathrm{~h}$ or $3 \mathrm{~h}$. Significant reduction in lever pressing was found in the 5 -min group $(n=6)$ and 1-h group $(n=7)$, but not in the oHFS alone group $(n=6)$ or 3-h group $(n=6)$ or vector-1-h control group $(n=6) .{ }^{*}$ indicates $P<0.05$; ${ }^{* *}$ indicates $P<0.001$; one-way repeated ANOVA $(\mathbf{g})$ and one-way ANOVA (h) followed by Tukey's multiple comparison test. i-k Similar to $\mathbf{f}$-h, but for fear training with oHFS 5 min and $1 \mathrm{~h}$ after the foot-shock. Significant reduction in lever pressing was found in the 5 -min group $(n=7)$ but not in the 1-h group $(n=6)$. The same data for the oHFS alone group in $\mathbf{g}$ - $\mathbf{h}$ is shown here again as a control. ${ }^{* * *}$ indicates $P<0.001$; one-way repeated ANOVA (j) and one-way ANOVA (k) followed by Tukey's multiple comparison test

recent study [8], Nabavi et al. used optogenetic stimulation to axonal inputs into the LA from the auditory cortex $(\mathrm{AC})$ and the medial geniculate nucleus (MGN), to investigate its association with the US (foot-shock) and how this association could be erased by long-term depression (LTD) and reestablished by long-term potentiation (LTP). Inspired by this paradigm, we expressed a light-activated channelrhodopsin ChIEF via adeno associated virus (AAV) in the AC and the MGN of SpragueDawley rats, and implanted an optic fiber above the LA for optogenetic stimulation of axonal inputs in this area from the virally infected neurons (Fig. 1a). Optogenetic stimulation and foot-shocks were used for association training, followed by fear recall testing with a pre-trained lever-press task $24 \mathrm{~h}$ later $[9,10]$ (Fig. 1b, Additional file 1: Materials and Methods).

With this system, we first confirmed that optogenetic moderate-frequency stimuli (oMFS; $10 \mathrm{~Hz}$ for $20 \mathrm{~s}$ ) paired with foot-shocks delivered at the end of each stimulation train (repeated every 3 min for 5 times) resulted in associative fear memory, which when recalled by a 2 -min train of $10 \mathrm{~Hz}$ optogenetic test stimuli (OTS) the next day, interrupted lever-press behavior (Fig. 1c-e). In contrast, "unpaired" oMFS with foot-shocks delivered at least 1-min afterwards could not establish such association: OTS the next day did not affect lever-press behavior (Fig. 1c-e). These results are consistent with previous findings [8], and suggest that optogenetic stimulation to the LA input projections could be used as an artificial "CS" for classical fear conditioning.

To evaluate the effects of a stronger artificial "CS" for potential "distant association", we chose optogenetic highfrequency stimuli (oHFS; $100 \mathrm{~Hz}$ light pulses for $1 \mathrm{~s}$, repeated 5 times every $3 \mathrm{~min}$ ), followed by a single 3 -s foot-shock with a delay of $5 \mathrm{~min}, 1 \mathrm{~h}$ or $3 \mathrm{~h}$ after the oHFS (Fig. 1f). In the lever-pressing test the next day, the same $10 \mathrm{~Hz}$ OTS was used because it is closer to the physiological firing frequency of LA neurons during natural fear recall [11]. Intriguingly, animals trained at 5- min and 1-h (but not 3-h) oHFS-shock intervals exhibited apparent fear recall behavior, with lever press significantly inhibited by OTS during the test (Fig. 1g-h). Therefore, a stronger CS-like stimulus could indeed establish distant association with the US delivered even after $1 \mathrm{~h}$ later.

As a control experiment, we used rats with AAV-hSyntdTomato injected in the AC and the MGN. These animals were trained with oHFS followed by foot-shock $1 \mathrm{~h}$ later (Fig. 1g-h, vector-1-h group). No fear response was detected during fear test the next day in these animals, in contrast to the significant reduction of lever pressing in the oChIEF expressing 1-h group (Fig. 1g-h). Thus, the behavioral responses are most likely due to optogenetically evoked neuronal activity in the LA. In another control experiment, we trained the animal using a similar paradigm but replaced the oHFS with oMFS $(10 \mathrm{~Hz}$ for $50 \mathrm{~s}$, total 500 pulses to match the pulse number in oHFS). We found that even when the foot-shock followed the prolonged oMFS by only $10 \mathrm{~s}$, no fear response was recalled by OTS the next day (Additional file 1: Fig. S1). Thus, the high stimulation frequency in oHFS was crucial for such distant association.

We further tested "backward" distant association by delivering the foot-shock first, followed by the oHFS at different intervals (Fig. 1i). Intriguingly, fear response was observed in the lever-pressing test the next day for animals with 5-min shock-oHFS interval but not those with 1-h interval (Fig. 1j-k). Thus, the distant association appeared to be bi-directional and temporally asymmetric, reminiscent of bi-directional trace conditioning observed in previous studies, although the latter has a much shorter timescale [12].

How could the oHFS form distant association with the foot-shock? It has been well established that synaptic plasticity such as NMDA receptor-dependent LTP in the LA plays an important role in fear conditioning $[8,13,14]$. With systemic NMDA receptor blocker MK801 injection before association training, we found that distant association at 1-h oHFS-shock interval could not be formed 
(Additional file 1: Fig. S2), indicating that NMDA receptor and activity-dependent synaptic plasticity are likely involved in this process. However, neuronal activation and NMDA receptor signaling in the current paradigm occur on the timescale of milliseconds to seconds, much shorter than that for distant association. One possible scenario is that the oHFS caused long-lasting changes in neuronal excitability and network activation, which act as a long "trace" to couple with the distant US. Along this line, comparing how different patterns of optogenetic stimuli elicit different levels of acute and chronic neural activation in the LA in vivo and in vitro could provide important insights. Another possibility is that the oHFS (perhaps via strong activation of NMDA receptors) initiated synaptic plasticity signals that lasted for minutes to hours and "consolidated" by subsequent US, through a mechanism analogous to "synaptic tagging" [15]. Whereas these possibilities remain to be investigated in future studies, the existence of distant association beyond the usual timescale of classical conditioning demonstrates the complexity of associative learning, and suggests new mechanisms underlying learning and memory as well as related brain disorders.

\section{Supplementary information}

Supplementary information accompanies this paper at https://doi.org/10. 1186/s13041-020-00587-4

Additional file 1: Materials and Methods, Fig. S1 Distant association could not be formed with oMFS followed by foot-shock, Fig. S2 Systemic administration of MK801 before fear training blocked distant association.

\section{Abbreviations}

CS: Conditioned stimulus; US: Unconditioned stimulus; AC: Auditory cortex MGN: Medial geniculate nucleus; LA: Lateral amygdala; oMFS: Optogenetic moderate-frequency stimuli; OHFS: Optogenetic high-frequency stimuli; OTS: Optogenetic test stimuli; NMDA: N-methyl-D-aspartate; LTP: Long-term potentiation; LTD: Long-term depression

\section{Acknowledgements}

We thank Dr. Bong-Kiun Kaang for helpful discussion.

\section{Authors' contributions}

P.-M.L. and G.-Q.B. supervised the research. F.L. conceived and designed the experiments. F.L., C.-H.J. and J.H. performed the experiments and analyzed the data. F.L., P.-M.L. and G.-Q.B. interpreted the data and wrote the paper. All authors read and approved the final manuscript.

\section{Funding}

This study was supported by grants from the National Natural Science Foundation of China (31630030 and 31070935) and the Strategic Priority Research Program of Chinese Academy of Science (XDB32030200).

\section{Availability of data and materials}

All data presented are available upon request.
Consent for publication

Not applicable.

\section{Competing interests}

The authors declare that they have no competing interests.

\section{Author details}

${ }^{1}$ CAS Key Laboratory of Brain Function and Disease, and School of Life Sciences, University of Science and Technology of China, Hefei 230026, Anhui, China. ${ }^{2}$ CAS Center for Excellence in Brain Science and Intelligence Technology, University of Science and Technology of China, Hefei 230026, Anhui, China.

Received: 20 January 2020 Accepted: 11 March 2020

Published online: 20 March 2020

\section{References}

1. Maren S. Neurobiology of Pavlovian fear conditioning. Annu Rev Neurosci. 2001;24:897-931.

2. Kim JJ, Jung MW. Neural circuits and mechanisms involved in Pavlovian fear conditioning: a critical review. Neurosci Biobehav Rev. 2006;30(2):188-202.

3. Mcalonan GM, Wilkinson LS, Robbins TW, Everitt BJ. The effects of Ampainduced lesions of the Septohippocampal cholinergic projection on aversive-conditioning to explicit and contextual cues and spatial-learning in the water maze. Eur J Neurosci. 1995;7(2):281-92.

4. Misane I, Tovote P, Meyer M, Spiess J, Ogren SO, Stiedl O. Time-dependent involvement of the dorsal hippocampus in trace fear conditioning in mice. Hippocampus. 2005;15(4):418-26.

5. Kwapis JL, Jarome TJ, Schiff JC, Helmstetter FJ. Memory consolidation in both trace and delay fear conditioning is disrupted by intra-amygdala infusion of the protein synthesis inhibitor anisomycin. Learn Mem. 2011 18(11):728-32.

6. Gilmartin MR, Kwapis $\mathrm{JL}$, Helmstetter FJ. Trace and contextual fear conditioning are impaired following unilateral microinjection of muscimol in the ventral hippocampus or amygdala, but not the medial prefrontal cortex. Neurobiol Learn Mem. 2012;97(4):452-64.

7. Kochli DE, Thompson EC, Fricke EA, Postle AF, Quinn JJ. The amygdala is critical for trace, delay, and contextual fear conditioning. Learn Mem. 2015; 22(2):92-100.

8. Nabavi S, Fox R, ProulX CD, Lin JY, Tsien RY, Malinow R. Engineering a memory with LTD and LTP. Nature. 2014;511(7509):348-52.

9. Repa JC, Muller J, Apergis J, Desrochers TM, Zhou Y, LeDoux JE. Two different lateral amygdala cell populations contribute to the initiation and storage of memory. Nat Neurosci. 2001:4(7):724-31.

10. Amorapanth P, Nader K, LeDoux JE. Lesions of periaqueductal gray dissociate-conditioned freezing from conditioned suppression behavior in rats. Learn Mem. 1999;6(5):491-9.

11. Grewe BF, Grundemann J, Kitch LJ, Lecoq JA, Parker JG, Marshall JD, et al. Neural ensemble dynamics underlying a long-term associative memory. Nature. 2017:543(7647):670

12. Quinn JJ, Oommen SS, Morrison GE, Fanselow MS. Post-training excitotoxic lesions of the dorsal hippocampus attenuate forward trace, backward trace, and delay fear conditioning in a temporally specific manner. Hippocampus. 2002;12(4):495-504.

13. Doyere V, Schafe GE, Sigurdsson T, LeDoux JE. Long-term potentiation in freely moving rats reveals asymmetries in thalamic and cortical inputs to the lateral amygdala. Eur J Neurosci. 2003;17(12):2703-15.

14. McKernan MG, Shinnick-Gallagher P. Fear conditioning induces a lasting potentiation of synaptic currents in vitro. Nature. 1997:390(6660):607-11.

15. Frey U, Morris RGM. Synaptic tagging and long-term potentiation. Nature. 1997;385(6616):533-6.

\section{Publisher's Note}

Springer Nature remains neutral with regard to jurisdictional claims in published maps and institutional affiliations. 